\title{
Fingerprint Databases for Theorems
}

\section{Sara C. Billey and Bridget E. Tenner}

"Fingerprint, in the anatomical sense, is a mark made by the pattern of ridges on the pad of a human finger. The term has been extended by metaphor to anything that can uniquely distinguish a person or object from another" [26].

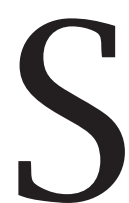

uppose that $M$ is a mathematician and that $M$ has just proved theorem $T$. How is $M$ to know if her result is truly new, or if $T$ (or perhaps some equivalent reformulation of $T$ ) already exists in the literature? In general, answering this question is a nontrivial feat, and mistakes sometimes occur.

Certain mathematical results have canonical representations, or fingerprints, and some families of fingerprints have been collected into searchable databases. If $T$ is such a theorem, then $M$ 's search will be greatly simplified. Note that the searchable nature of a database is important here. An analogue of "alphabetical order" does not exist for all structures, and so it is important that $M$ be able to query the fingerprint of $T$ instead of needing to browse through all existing catalogued results.

Sara C. Billey is professor of mathematics at the University of Washington in Seattle. Her email address is bi11ey@math. washington. edu.

Bridget E. Tenner is associate professor of mathematics at DePaul University in Chicago. Her email address is bridget@math. depau1 .edu.

Research of the first author was partially supported by a grant from the National Science Foundation DMS-1101017.

Research of the second author was partially supported by a DePaul University Competitive Research Leave grant.

DOI: http://dx.doi.org/10.1090/noti1029
A revolutionary mathematical tool appeared online in 1996-Neil Sloane's collection of integer sequences, along with mathematical interpretations of the numbers, formulas for generating them, computer code, references, and relevant links. This was the On-Line Encyclopedia of Integer Sequences (OEIS) [22], originally hosted on Sloane's website at AT\&T Labs. Anyone with access to the Internet could peruse the database, and anyone could submit a sequence or supplemental data to the database. All for free. Thanks to Sloane's tireless efforts and a worldwide community of contributors, the collection has grown to well over 200,000 sequences to date, drawing results from all areas of mathematics. The sequences in the OEIS act as fingerprints for their associated entries, the majority of which encode mathematical statements. While the fingerprints in the OEIS have a specific input structure, the sequences can arise in many contexts, including arrays of data, coefficients of polynomials, enumeration problems, subway stops, and so on. The OEIS itself is the database for these fingerprints. The impact on research is clearly established by over 3,000 articles to date citing the OEIS [23].

Fingerprinting has made an impact in many scientific fields. For example, fingerprinting documents is crucial in computer science for reducing duplication in Web search results, isotopic fingerprints are used in fields ranging from chemistry to archaeology, and there is of course extensive use of fingerprinting in forensic science.

There are other families of mathematical results that have their own identifying fingerprints, not in the form of integer sequences. Searchable catalogues are already in use for some of these families, 
while no such directories yet exist for others. The aim of this article is to give these resources greater exposure and also to encourage the community to create and support new fingerprint databases for other mathematical structures. Note that what we propose is not simply enhanced digital mathematical resources. Rather, a fingerprint database of theorems should be a searchable, collaborative database of citable mathematical results indexed by small, language-independent, and canonical data.

Every day new tools for searching the scientific literature are established. To be clear, this article will be out of date the moment it is published. In fact, active research at the intersection of mathematics, computer science, and linguistics is devoted to organizing mathematics into more searchable formats, including the Mathematical Knowledge Management and Intelligent Computer Mathematics conferences. An example outside of mathematics is biomedical natural language processing, known as BioNLP [11]. We expect that, one day, natural language processing will be applicable to theorems and will significantly facilitate M's search through the literature. The question is, what can we do until then?

Rod Brooks and his group at MIT used the phrase

"fast, cheap, and out of control"

to describe an emphasis on building small, cheap, and redundant robots instead of overly complex single machines [6]. We suggest that a similar approach to fingerprinting theorems can make a big impact in the near future, while more finessed tools are being developed in the background. It is better to start a theorem collection now-with an imperfect but efficient fingerprint-than to waste time awaiting an epiphany about the perfect mechanism for encoding this data.

In a sense, we are proposing a new line of research for mathematicians to address: what are the fingerprintable theorems within each discipline of mathematics, and what might those fingerprints look like?

\section{Known Results Can Be Hard to Find}

Theorems are usually written in human-readable language. They employ specialized vocabulary, functions, and layers of hypotheses and implications. A theorem in one branch of mathematics can resurface in another context, and the two statements may bear little superficial resemblance to each other. Search engines can help uncover a result if it is accessible online and there is a name associated with it, such as for a solution to a famous conjecture, in which case the name, or names, would be the fingerprint. For example, one can easily ask a search engine for information about Fermat's Last Theorem, which would lead $M$ to discover that her result $T$ was already proved by Wiles [28].

Formulas are prevalent in mathematical research but are inherently difficult to query. For example, $M$ would have to make decisions about notation, variable names, and formatting. Moreover, even if search engines did have a good mechanism for querying formulas, it might not be especially useful-a given formula can often be stated in a variety of ways. For example, the following basic trigonometric identities are equivalent:

$$
\begin{aligned}
& \sin ^{2} \theta+\cos ^{2} \theta=1, \\
& 2 \tan ^{2} \theta+2=2 \sec ^{2} \theta,
\end{aligned}
$$

and

$$
3+3 \cot ^{2} \theta=3 \csc ^{2} \theta .
$$

If our mathematician $M$ has discovered a new statement of an existing formula, a search engine might have difficulty detecting that her result is equivalent to the known one.

There have been many ideas put forth for improving the search tools for formulas in the literature. In fact, search tools themselves can contribute to mathematical results. Notably, Gödel invented a numerical encoding of formulas as a step toward proving his famous Incompleteness Theorem [12]. However, the procedure is not unique and it is certainly not efficient. For example, the Gödel number of the formula " $0=0$ " is $2^{6} \times 3^{5} \times 5^{6}=243,000,000$. More recently, Borwein and Macklem addressed the question of how best to add hyperlinks to electronically available textbooks [4].

Of course, $M$ 's search through existing literature for any hint of her theorem $T$ would have been much harder prior to the Internet. There are examples throughout mathematical history of theorems having been discovered, and subsequently rediscovered independently-sometimes over and over again. For example, the characterization of higher-dimensional regular polytopes, attributed to Schläfli, had been recovered at least nine other times by the end of the nineteenth century [9].

\section{Benefits of a Good Fingerprint Database}

We wish to proselytize for the accumulation of theorem fingerprints into databases. We urge the reader to become a collector-a connoisseur, even!

First, though, we must explain how the OEIS encodes theorems - after all, its primary purpose is to collect and catalogue integer sequences. In fact, the theorems can be found within the architecture of this database-namely, by the inclusion of 
other fields associated with each sequence such as "name", "comments", "formula", and so on.

If our mathematician $M$ is going to make use of the OEIS, it is because she has encountered a sequence of integer data within her work. Then $M$ runs a query against the OEIS, using her data. Even a relatively small subsequence-perhaps just two numbers - can sometimes determine a unique entry in the OEIS. The responses from M's search enable her to connect her data to known literature, to find formulas, to make conjectures, and so on.

For example, if $M$ enters $0,1,1,2,3,5,8,13,21$ into the OEIS, the first option it returns is sequence A000045, the Fibonacci numbers. Two of the comments for this entry are

- $F(n+2)=$ number of subsets of $\{1,2$, $\ldots, n\}$ that contain no consecutive integers, and

- $F(n+1)=$ number of tilings of a $2 \times n$ rectangle by $2 \times 1$ dominoes.

Thus this entry encodes a variety of results, including the following.

Theorem ([22, A000045]). The subsets of $\{1,2, \ldots, n\}$ containing no consecutive integers are in bijection with the tilings of a $2 \times(n+1)$ rectangle by $2 \times 1$ dominoes, and these are each enumerated by the $(n+2) n d$ Fibonacci number.

In this way, each entry in the OEIS chronicles a mathematical theorem, and the integer sequence associated with the entry is that theorem's fingerprint. The OEIS is arguably the most established fingerprint database for theorems to date.

\section{Other Fingerprint Databases}

Depending on the structure of theorem $T$, the OEIS is not the only tool of its kind available to the curious $M$. We will describe some of the fingerprint databases for theorems that already exist in this section. These databases augment the classical approach to finding theorems in the literature, including books, journals, MathSciNet, the arXiv, and the World Digital Mathematics Library.

\section{Permutation Patterns}

The Database of Permutation Pattern Avoidance (DPPA) [24] contains collections of permutationsthought of as patterns - whose avoidance exactly characterizes particular phenomena. The second author started this database in 2005, and it has grown to more than forty sets of patterns so far. In addition to the patterns themselves, each entry in the DPPA includes the phenomenon (or phenomena) being characterized, references to existing literature, and a link to the OEIS whenever possible. The DPPA is searchable both by permutation (pattern) and by keyword.
For example, if theorem $T$ involves permutations avoiding the two patterns 3412 and 4231, then the DPPA would have directed $M$ to entry P0005, for the set $\{3412,4231\}$. The two descriptions for this entry are

- permutations with rank symmetric order ideals in the Bruhat order and

- permutations indexing smooth Schubert varieties,

as described in [7], [17].

Each entry of the DPPA represents a characterization theorem. The theorem for the entry just described would be as follows.

Theorem ([24, P0005]). The permutations with rank symmetric order ideals in the Bruhat order are exactly those that index smooth Schubert varieties, and they are precisely the permutations that avoid the patterns 3412 and 4231.

The fingerprint for each DPPA theorem is its associated set of patterns, and the DPPA itself is the database for these fingerprints.

\section{FindStat}

FindStat [3] is a database of statistics on combinatorial objects. It was created in 2011 by Berg and Stump and currently catalogues over one hundred statistics. If $M$ has obtained some data about one of these objects, then she could enter her data into FindStat, and it would tell her if this particular statistic is included in the database. If so, FindStat would identify the standard vocabulary used for that statistic. This would equip $M$ with searchable terminology, allowing her to discover any relevant existing literature.

\section{Hypergeometric Series}

Every hypergeometric series can be written in a canonical form, and this form serves as the fingerprint for these objects. It has long been common to store identities for these series in tables, listed in a given order by these canonical forms. For example, Bailey published such a collection in 1935 [2]. Perhaps this book is the original fingerprint database for theorems.

The modern approach has taken research in hypergeometric identities one step further. The WZ method for finding identities involving hypergeometric series has been described in the book $A=B$ by Petkovšek, Wilf, and Zeilberger [19]. Using these algorithms, one can determine definitively if a hypergeometric series has a closed form or not. If there is a closed form, then the WZ method will produce it, given enough computational time and memory. Furthermore, this procedure will give a proof certificate that can be used to check the identity. Many new identities and new proofs 
of known identities have been found using the WZ method, for example [10]. What this resource currently lacks is a way to connect results to existing literature, pointing our mathematician $M$ to what is already known about each identity.

The National Institute of Standards and Technology's (NIST) Digital Library of Mathematical Functions (DLMF) also includes many hypergeometric identities indexed by canonical form and some references. We should point out, however, that neither the WZ method nor the DLMF form a fingerprint database for theorems themselves in their current form. Perhaps there could be a collaborative effort to catalogue all known hypergeometric identities with extensive references and entries searchable by their canonical forms. If so, all new identities found by the WZ method could include their proof certificate as a comment. This could provide a useful place to "publish" proof certificates.

\section{Constructing a Fingerprint Database Is Not Always Easy}

An important asset of the OEIS, the DPPA, FindStat, and the WZ method is that the fingerprints they use are language independent. More precisely, their input is entirely numerical and canonical-free from specialized vocabulary. This seems to be a necessary feature of a good fingerprint database for theorems.

Another desirable feature of a productive fingerprint database is that it should reference existing literature whenever possible. Cross-references within a single database and between different databases can only enhance the state of knowledge. Features like computer code and external links can be highly beneficial when relevant. For example, any integer sequence associated with a theorem in a new fingerprint database should reference the relevant OEIS entry.

Because mathematics is so broad and develops so quickly, a fingerprint database for theorems should be collaborative-publicly available and welcoming additions from anyone, subject to editorial standards. The Wikipedia model for an open database is a highly successful model of this idea. However, one does not need to learn MediaWiki before starting a collection of theorem fingerprints; rather, one could simply ask for new database entries to be submitted in some kind of standard format which can easily be added to the database.

Finally, it is most convenient for the fingerprint to be encoded in a small amount of data. There is a natural conflict between keeping fingerprints small and uniquely identifying each object in the database. Certainly some compromises to one or both of these might be necessary. An efficient fingerprint encryption may be permitted to return some false positives, but it should never return a false negative. The possibility of false positives is all the more reason for additional fields within the database entries, to distinguish the true from the false positives. For example, querying the first nine Fibonacci numbers will return many false positives in the OEIS, but $M$ can weed through them by reading through their full records.

There are certainly some challenges to creating a fingerprint database for theorems. These include identifying the right data structure as the fingerprint, determining a canonical format, addressing structures that have no obvious numerical data, and compactly encoding a given fingerprint. We hope these obstacles will not be too daunting, though, because an imperfect resource is still better than no resource at all. Two examples are given below.

\section{Example: Fingerprinting Graphs}

Theorems about finite graphs deserve a fingerprint database. There exist numerous classification theorems in graph theory that equate graph containment with important properties. One of the monumental results of the twentieth century is the Graph Minor Theorem by Robertson and Seymour [21]:

Any family $\mathcal{F}$ of graphs that is closed under taking minors can be characterized as the set of all graphs whose minors avoid a finite list $L(\mathcal{F})$.

This result certainly suggests that graphs can fingerprint theorems. The Wagner formulation of Kuratowski's Theorem is an example of this situation [16], [25]:

A simple graph $G$ is planar if and only if $G$ has no minor isomorphic to the graphs known as $K_{3,3}$ and $K_{5}$.

Graphs arise as classification tools in many fields of mathematics, including Hales's proof of Kepler's Conjecture [14] and the classification of finite Coxeter groups [15, Chapter 2 and Section 6.4].

One could enumerate the results of a graph theorem, say, by counting the graphs of each size possessing a certain property. The resulting sequence could be an entry in the OEIS. However, a graph theorem database would still be relevant because it could track more specific graph properties through further refinement and cataloguing. Moreover, and perhaps more persuasively, counting graphs is not an easy computational problem, so this partial enumerative fingerprint would not uniquely identify the appropriate entry in the OEIS. For example, the linklessly embeddable graphs in Euclidean space are characterized by avoiding the Petersen family of graphs, which include seven 
graphs having between six and ten vertices each. It is computationally infeasible to compute the number of linklessly embeddable graphs on six, seven, eight, nine, and ten vertices, which would be the first few times at which this sequence differs from the sequence enumerating all graphs.

There currently exist many online resources for graph data, such as House of Graphs [5] and the tools listed at [27]. However, none of these resources is a database of theorems (at present). It is inherently difficult to fingerprint graph theorems using searchable, canonical, and concise numerical data. In particular, there is not an obvious choice for the best way to fingerprint a graph.

The adjacency matrix of a graph describes the graph uniquely in numerical data. Often in graph theory, a classification theorem depends only on isomorphism classes. This could pose a problem if the fingerprint of a graph is its adjacency matrix, because isomorphic graphs can have different adjacency matrices. For example, the graph with two adjacent vertices and one isolated vertex could be represented by any of

$$
\left[\begin{array}{lll}
0 & 1 & 0 \\
1 & 0 & 0 \\
0 & 0 & 0
\end{array}\right],\left[\begin{array}{lll}
0 & 0 & 1 \\
0 & 0 & 0 \\
1 & 0 & 0
\end{array}\right] \text {, and }\left[\begin{array}{lll}
0 & 0 & 0 \\
0 & 0 & 1 \\
0 & 1 & 0
\end{array}\right] \text {. }
$$

We can, of course, handle this difficulty by choosing a canonical representative in each isomorphism class, such as the adjacency matrix whose row reading word is smallest in lexicographic order. However, finding such a canonical adjacency matrix is no easy task: there is no known polynomial time algorithm for testing graph isomorphism. In fact, it is an open question whether the graph isomorphism problem is NP-complete.

Degree sequences are an attractive choice for fingerprints because they are much easier to encode than adjacency matrices. If one were to fingerprint graph families by lists of degree sequences written in lexicographic order, then $K_{3,3}$ and $K_{5}$ would be encoded as the list $[[3,3,3,3,3,3],[4,4,4,4,4]]$. Querying this list in a database of graph theorems, the mathematician $M$ would learn that these two graphs are related to planar graphs via Kuratowski's Theorem.

On the other hand, a degree sequence does not determine a unique graph. For example, both

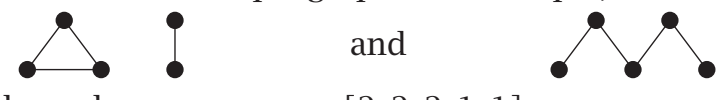

have degree sequence $[2,2,2,1,1]$.

Theorems about specific graphs or families of graphs may be rare enough that the compromises one makes when fingerprinting by degree sequences might not result in too many false positives. Indeed, as we have said before, it is better to have a collection of theorems with an imperfect fingerprint than to have no collection at all!

\section{Example: Finite Groups}

The finite simple groups have been completely classified [29]. These groups fall into six families, and the title for each group is given by a combination of letters and numbers. For example, one group is denoted ${ }^{3} D_{4}\left(q^{3}\right)$. These groups, and various details about them, are collected in the ATLAS of Finite Group Representations [1]. To date, this resource includes more than 5, 000 representations of more than 700 groups.

The current implementation of the ATLAS does not allow users to search the database by numerical invariants of the groups; thus it is not a fingerprint database as we have defined it. To find the details of a group, one must know its title or something about where it fits into the classification.

To make the ATLAS into a fingerprint database, one would have to add a feature where groups could be detected by some numerical invariant(s). For example, an additional search box could be added to the main webpage to access the database by entering the order of a group. Then the order would act as the fingerprint. There are groups of the same order already in the database, but perhaps the number of coincidences is small enough that a user could prune the results via the many other entries available. Additional invariants might also be used to refine the search.

\section{What Should Happen Next}

We believe that many families of theorems can be fingerprinted-some identified by obvious data structures, others perhaps by less obvious structures. We encourage everyone in the mathematical community to look at their own work for results that can be identified by some form of compact data. In fact, any structure that has a canonical parameterization merits this attention. Additionally, a long-term benefit of having these databases is that structures amenable to fingerprinting may also be amenable to computer proof verification systems and learning algorithms, as with the Four Color Theorem [13], [20] and permutation patterns [18].

Clever insight, beyond what is currently common practice, might be necessary to find an appropriate fingerprint. In fact, the need to find theorem fingerprints can drive future research.

Many disciplines of mathematics would benefit from the greater context of a theorem database. The accessibility of mathematical research in the last few decades has flourished. In the past few years alone, we have seen substantial growth as measured in mathematics articles posted on the arXiv, increasing from 4,654 articles in 2002 to 24,176 articles in 2012 [8]. With this level of productivity, fingerprint databases are even more 
valuable. These resources-both the ones that currently exist and those that we hope the readers will create-enhance experimental mathematics, help researchers make unexpected connections between areas of mathematics, and even improve the refereeing process. We encourage everyone to follow Neil Sloane's lead and to take up such a collection.

\section{Hats off to Neil!}

\section{Acknowledgments}

First and foremost, we want to thank the OEIS and all of its contributors, with special thanks to Neil Sloane. We also thank all the contributors to the other resources we have referenced and used in our own work. We would like to thank Chris Berg, Jon Borwein, Neil Calkin, Chris Godsil, Ron Graham, Ursula Martin, Brendan Pawlowski, Christian Stump, Jair Taylor, Lucy Vanderwende, Paul Viola, and Doron Zeilberger for helpful discussions while preparing this article. We thank the organizers of the ICERM workshop on Reproducibility in Computational and Experimental Mathematics for presenting a chance to discuss these ideas with a broad community. Finally, the first author thanks Rod Brooks for the opportunity to work in his lab as an undergraduate at the height of the "fast, cheap, and out of control" revolution in robotics.

\section{References}

[1] R. Abbott, J. Bray, S. Linton, S. Nickerson, S. NORTON, R. PARKer, I. SUleiman, J. TRIPP, P. WALSH, and R. WILSON, ATLAS of Finite Group Representations, published electronically at http://brauer .maths.qmu1 .ac.uk/At1as/v3/ March 14, 2013.

[2] W. N. BAILEY, Generalized Hypergeometric Series, Cambridge Tracts in Mathematics and Mathematical Physics, No. 32, Cambridge University Press, Cambridge, 1935.

[3] C. Berg and C. STUMP, FindStat, published electronically at http://www.findstat. org/StatisticsDatabase, June 13, 2013.

[4] J. M. BORWEIN and M. MACKLEM, Retro-enhancement of Mathematical Literature, published electronically at http://docserver.carma.newcast7e.edu.au/ 339/, April 13, 2013.

[5] G. Brinkmann, J. Goedgebeur, H. Mélot, and K. CoOlSAET, House of Graphs: A database of interesting graphs, Discrete Appl. Math. 161 (2013), 311-314, published electronically at http://hog.grinvin. org, April 13, 2013.

[6] R. BRooks, Fast, cheap and out of control: A robot invasion of the solar system, J. British Interplanetary Society 42 (1989), 478-485.

[7] J. CARRELL, The Bruhat Graph of a Coxeter Group, a Conjecture of Deodhar, and Rational Smoothness of Schubert Varieties, Proc. Symp. Pure Math. 56 (1994), 53-61.
[8] Cornell University Library, Mathematics ArXiv, published electronically at http://arxiv.org/ archive/math, March 14, 2013.

[9] H. S. M. CoXeTER, Regular Polytopes, Methuen and Co., London, 1948.

[10] S. B. EKHAD and D. ZEILbERGER, A WZ proof of Ramanujan's formula for $\pi$, Geometry, Analysis, and Mechanics, J. M. Rassias (ed.), World Scientific, Singapore, 1994, pp. 107-108.

[11] B. FUTRELLE, Natural language processing of biology text, published electronically at http://bion $1 p$. org/, April 10, 2013.

[12] K. GöDEL, Über formal unentscheidbare Sätze der Principia Mathematica und verwandter Systeme I, Monatsheft Math. Physik 38 (1931), 173-198.

[13] G. GONTHIER, Formal proof-the four-color theorem, Notices of the AMS 55 (2008), 1382-1393.

[14] T. C. HAlES, A proof of the Kepler conjecture, Ann. Math. 162 (2005), 1065-1185.

[15] J. HuMPHREYs, Reflection Groups and Coxeter Groups, Cambridge Studies in Advanced Mathematics 29, Cambridge University Press, 1990.

[16] K. KURATOWSKI, Sur le problème des courbes gauches en topologie, Fund. Math. 15 (1930), 271-283.

[17] V. LAKSHMIBAI and B. SANDHYA, Criterion for smoothness of Schubert varieties in $S L(n) / B$, Proc. Indian Acad. Sci. (Math. Sci.) 100 (1990), 45-52.

[18] H. MAgNuSSON and H. UlFARSSON, Algorithms for discovering and proving theorems about permutation patterns, arXiv:1211.7110.

[19] M. Petkovšek, H. Wilf, and D. Zeilberger, $A=B$, A. K. Peters, Wellesley, MA, 1996.

[20] N. Robertson, D. P. SAnders, P. Seymour, and R. Thomas, The four-color theorem, J. Combin. Theory Ser. B 70 (1997), 2-44.

[21] N. Robertson and P. D. Seymour, Graph Minors. XX. Wagner's Conjecture, J. Combin. Theory Ser. B 92 (2004), 325-357.

[22] N. J. A. SloAne, The on-line encyclopedia of integer sequences, published electronically at http:// oeis.org, March 14, 2013.

[23] , Works citing the OEIS, http://oeis.org/ wiki/Works_Citing_OEIS, March 22, 2013.

[24] B. E. TENNER, Database of permutation pattern avoidance, published electronically at http://math. depau1.edu/bridget/patterns.htm7, April 10, 2013.

[25] K. WAGNER, Über eine Eigenschaft der ebenen Komplexe, Math. Ann. 114 (1937), 570-590.

[26] Wikipedia contributors, Fingerprint (disambiguation), Wikipedia, The Free Encyclopedia, published electronically at http://en.wikipedia.org/wiki/ Fingerprint_(disambiguation), March 14, 2013.

[27] Wikipedia contributors, Graph database, Wikipedia, The Free Encyclopedia, published electronically at http://en.wikipedia.org/wiki/Graph_database, March 14, 2013.

[28] A. WILES, Modular elliptic curves and Fermat's Last Theorem, Ann. Math. 141 (1995), 443-551.

[29] R. WiLson, The Finite Simple Groups, Graduate Texts in Mathematics 251, Springer-Verlag, Berlin, 2009. 